\title{
Advancements in PARP1 Targeted Nuclear Imaging and Theranostic Probes
}

\author{
Ramya Ambur Sankaranarayanan ${ }^{1}$, Susanne Kossatz ${ }^{2,3,4}$, Wolfgang Weber ${ }^{2}$, \\ Mohsen Beheshti ${ }^{1,5}$, Agnieszka Morgenroth ${ }^{1}$ and Felix M. Mottaghy 1,6,* \\ 1 Department of Nuclear Medicine, University Hospital Aachen, RWTH Aachen University, \\ 52074 Aachen, Germany; rambursankar@ukaachen.de (R.A.S.); mbeheshti@ukaachen.de (M.B.); \\ amorgenroth@ukaachen.de (A.M.) \\ 2 Department of Nuclear Medicine, University Hospital Klinikum Rechts der Isar, Technical University \\ Munich, 81675 Munich, Germany; s.kossatz@tum.de (S.K.); w.weber@tum.de (W.W.) \\ 3 Central Institute for Translational Cancer Research (TranslaTUM), Technical University of Munich, \\ 81675 Munich, Germany \\ 4 Department of Chemistry, Technical University of Munich, 85748 Munich, Germany \\ 5 Department of Nuclear Medicine and Endocrinology, Paracelsus Medical University, 5020 Salzburg, Austria \\ 6 Department of Radiology and Nuclear Medicine, Maastricht University Medical Center (MUMC+), \\ 6202 Maastricht, The Netherlands \\ * Correspondence: fmottaghy@ukaachen.de; Tel.: +49-241-808-8741
}

Received: 11 June 2020; Accepted: 3 July 2020; Published: 6 July 2020

\begin{abstract}
The central paradigm of novel therapeutic approaches in cancer therapy is identifying and targeting molecular biomarkers. One such target is the nuclear DNA repair enzyme Poly-(ADP ribose) polymerase 1 (PARP1). Sensitivity to PARP inhibition in certain cancers such as gBRCA ${ }^{\text {mut }}$ breast and ovarian cancers has led to its exploitation as a target. The overexpression of PARP1 in several types of cancer further evoked interest in its use as an imaging target. While PARP1-targeted inhibitors have fast developed and approved in this past decade, determination of PARP1 expression might help to predict the response to PARP inhibitor treatment. This has the potential of improving prognosis and moving towards tailored therapy options and/or dosages. This review summarizes the recent pre-clinical advancements in imaging and theranostic PARP1 targeted tracers. To assess PARP1 levels, several imaging probes with fluorescent or beta/gamma emitting radionuclides have been proposed and three have advanced to ongoing clinical evaluation. Apart from its diagnostic value in detection of primary tumors as well as metastases, this shall also help in delivering therapeutic radionuclides to PARP1 overexpressing tumors. Henceforth nuclear medicine has now advanced towards conjugating theranostic radionuclides to PARP1 inhibitors. This paves the way for a future of PARP1-targeted theranostics and personalized therapy.
\end{abstract}

Keywords: PARP inhibition; PARP1 tracers; PARP1 theranostic probes; PET/SPECT imaging; Auger and Alpha emitters

\section{Introduction}

DNA damage is recognized and repaired specifically by different repair mechanisms [1]. One of the early sensors of DNA single strand breaks is Poly ADP-Ribose Polymerase 1 (PARP1), a nuclear protein. PARP1 and other 16 PARP family members function as a catalyst for Poly (ADP-Ribosylation) (PARylation) using Nicotinamide Adenine Dinucleotide (NAD+) as the ADP donor [2]. PARP1 recognizes strand breaks, binds to the DNA backbone, recruits acceptor proteins, post-translationally modifies them by transferring PAR polymers (PAR-ylation) and also undergoes auto-PAR- ylation [2,3].Importantly, in cases of defective double strand DNA damage 
repair mechanisms (homologous recombination), possibly due to mutated Breast Cancer 1/2 (BRCA1/2 ${ }^{\text {mut }}$ ) proteins, PARP1-mediated processes can take over the repair [2,4]. Unlike healthy cells, rapidly proliferating cancer cells are under higher replicative stress, which leads to genomic instability causing PARP1 overexpression. Hence, PARP1 is a critical protein, which has become an important target for inhibition therapies, especially in BRCA1/2 ${ }^{\text {mut }}$ patients. This scenario, where simultaneous loss-of-function/inhibition of two complementary proteins resulting in cytotoxicity, is termed "Synthetic Lethality". Till now, various PARP inhibitors (PARPis) such as olaparib (2014) [5], rucaparib (2016) [6], niraparib (2017) [7] and talazoparib (2018) have been clinically approved by the Food and Drug Administration (FDA) and European Medicine Agency (EMA) [8,9]. The additional use of DNA damaging agents might lead to an increased dependence on PARP1 activity for repair and by this would amplify a cells/tumors sensitivity to PARP inhibition. Combination therapies of PARPi with other DNA damaging therapies such as radiation therapy, chemotherapeutic drugs (e.g., Doxorubicin) [10] or anti-angiogenic therapy and immunotherapy are being assessed to improve cytotoxicity, and by this, the therapy efficacy and outcome as discussed in a recent review [11].

With high prominence of PARPi in cancer therapy, determining PARP1 expression levels in tumors might help to predict the sensitivity to PARP1-targeted therapy. PARP-imaging agents are potentially useful in the pre-treatment phase as a guidance to predict therapy response and to facilitate patient stratification, and in interim and post-treatment phases to quantify tumor response to therapy. Initially, fluorescent tagged olaparib derivatives were developed for optical imaging, one of which (PARPi-FL) has progressed to a clinical trial for oral cancer detection upon topical application. Report on the first in-human trial shows quantifiable PARPi-FL-based tumor detection in human tissue specimens and feasible application methods for esophageal tumor imaging upon topical application of PARPi-FL [12,13]. The need for depiction of PARP1 expression on the whole-body level initiated design and development of radiolabeled PARPi derivatives, which led to non-invasive determination of PARP1 expression by imaging modalities like Positron Emission Tomography (PET) or Single Photon Emission Computed Tomography (SPECT). Synthesis of radiolabeled PARP1-targeting imaging probes have been in an accelerated drive in this past decade. Radiohalogens such as ${ }^{18} \mathrm{~F},{ }^{123} \mathrm{I}$, and ${ }^{131} \mathrm{I}$ are favored for radiolabeling PARPis rather than radiometals $\left({ }^{68} \mathrm{Ga},{ }^{99 \mathrm{~m}} \mathrm{Tc}\right)$ due to their ease of radiolabeling without the need of a chelator, apart from their favorable physical characteristics [14]. Nevertheless, there is a need to summarize and identify the advantages to determine the most relevant application and suitable candidates for clinical application. Hence, the purpose of this review is to compile and update on important PARP1-targeted radio-theranostics developed recently, in the context of their specific applications in cancer diagnosis and therapy. Figure 1 illustrates the different approaches in PARP mediated therapeutic, diagnostic and theranostics. 


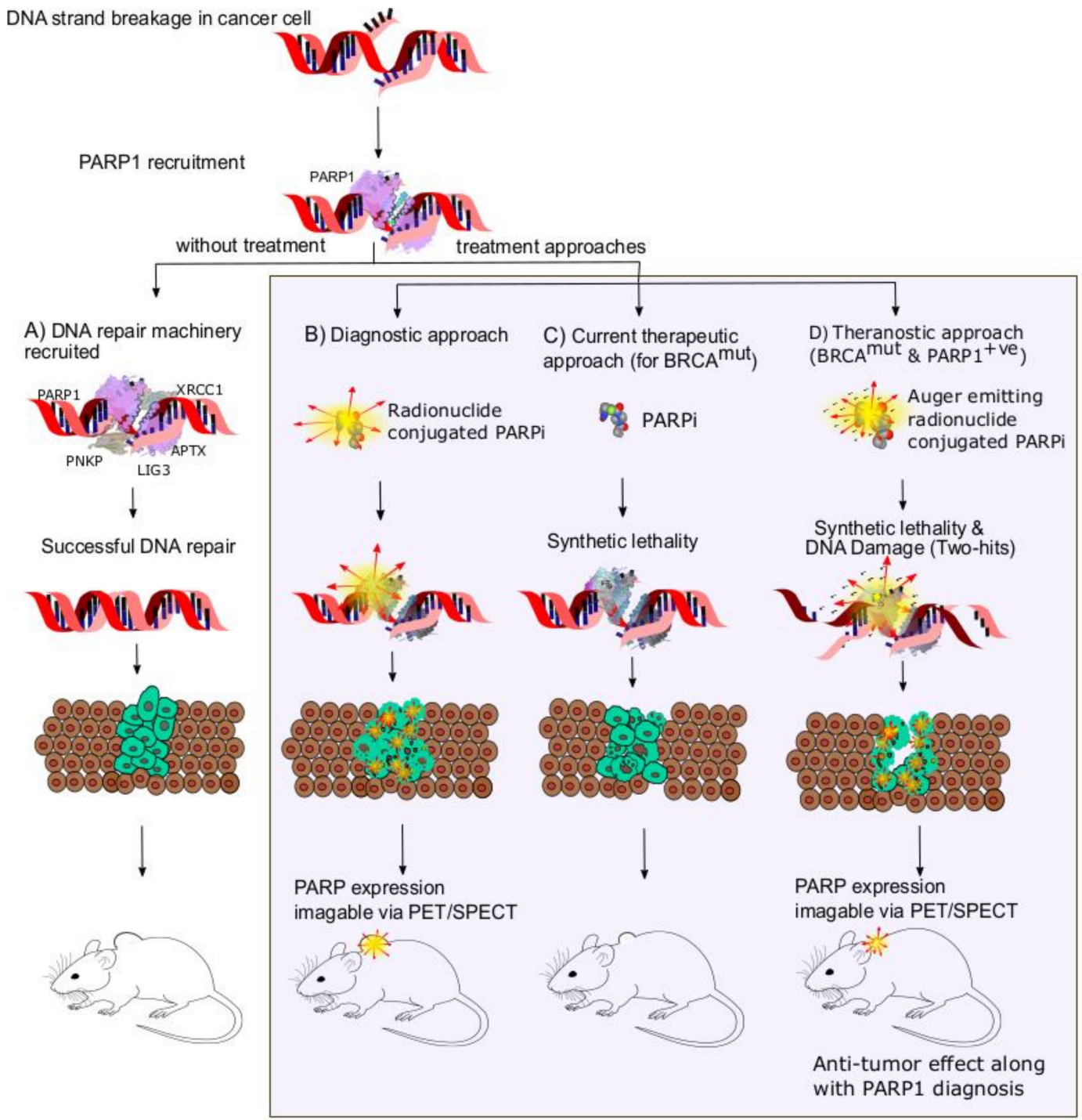

Figure 1. Schematic representation of PARP1-targeted therapy and imaging approaches. (A) Upon no treatment, PARP1-mediated repair enables cancer cell proliferation and tumour growth. (B) PET/SPECT radionuclide-conjugated PARP inhibitors enable imaging of the tumour tissues and PARP1-expression levels. (C) PARP inhibitor-based targeted therapy causes "Synthetic lethality", thereby inhibiting DNA repair mechanisms. (D) Auger electron (displayed in picture), Beta or Alpha particle-emitting radionuclides are able to cause DNA damage apart from synthetic lethality, and functions as a "two-hit" strategy and enhances apoptosis of cancer cells. For optimal synthetic lethality, supplementation with PARP inhibitors would be required.

\section{Pharmacokinetic Considerations}

Clinically approved PARPi core structures or their derivatives have been used for labelling with radionuclides (Table 1). Ideally, pharmacokinetic defining parameters like the parent drug's molecular weight (MW), charge, serum stability, vascular retention ( $\%$ plasma protein binding/\%PPB), lipophilicity ( $\log \mathrm{P}_{\mathrm{oct}}$ or $\log \mathrm{P}_{\mathrm{CHI}}$ values), affinity $\left(\mathrm{IC}_{50}\right)$, and PARP1 specificity should remain largely unchanged. These parameters will influence the in vivo behaviour such as tumor uptake and target to background ratios (TBR). Most reported radiotracers have shown an increase in lipophilicity and a predominantly hepatobiliary clearance. For example, the radionuclide conjugation of olaparib elevated lipophilicity for olaparib derivatives ${ }^{18} \mathrm{~F}-20$ and ${ }^{18} \mathrm{~F}-\mathrm{PARP}$ from $\log \mathrm{P}_{\text {oct }}=1.95$ to Log $\mathrm{P}_{\text {oct }}=2.51$ and $\log P_{C H I}=2.15$, respectively $[15,16]$. 
Table 1. Summary of the PARP tracers, their respective precursors, modality of imaging, and their current stage of development. Chemical structures of parent PARP inhibitors and their derivative radiotracers show structural modifications (green) and radionuclides (red). Modality of Imaging shows their PET (Positron Emission tomography)/SPECT (Single photon emission computed tomography) tracer capability.

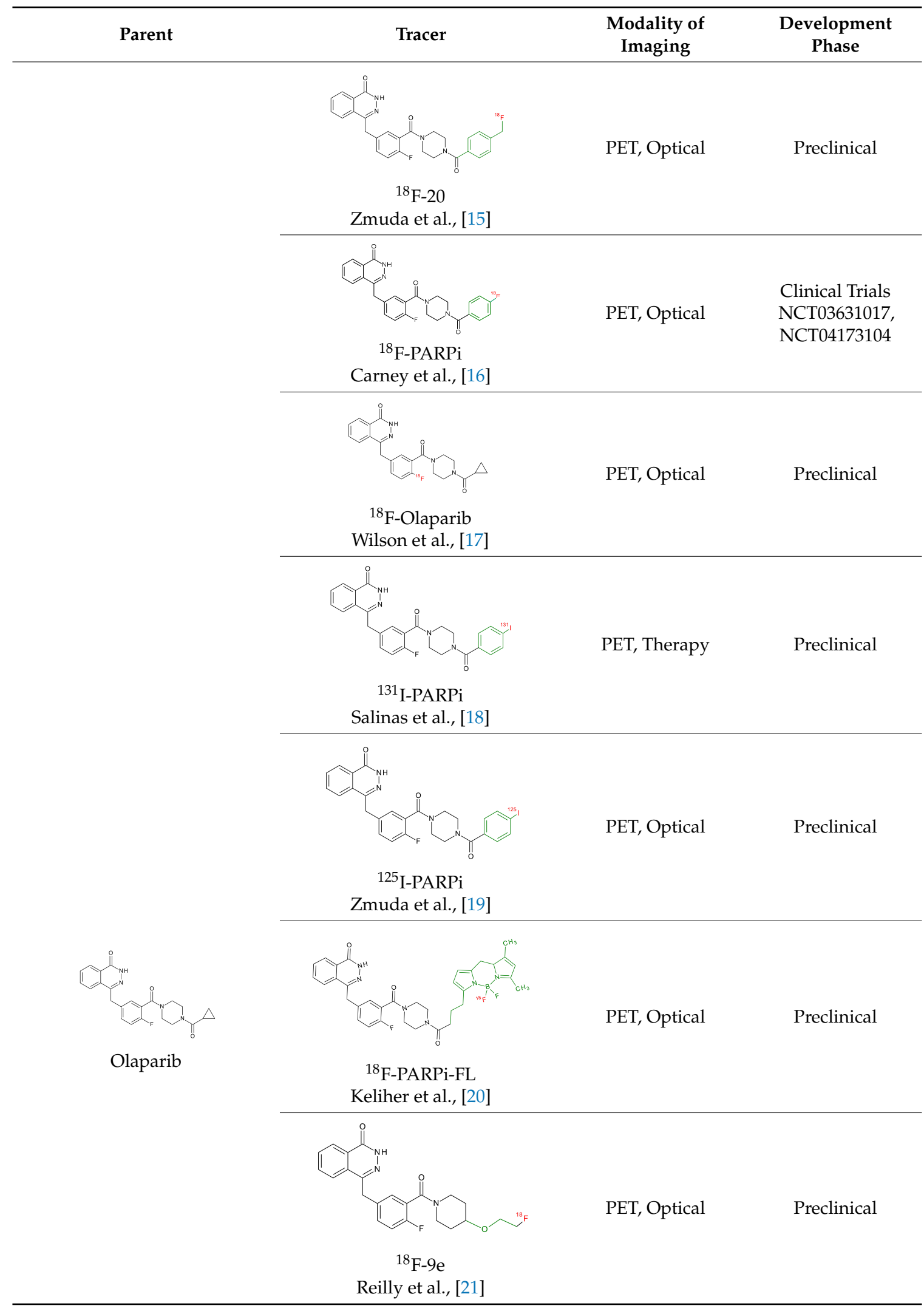


Table 1. Cont.

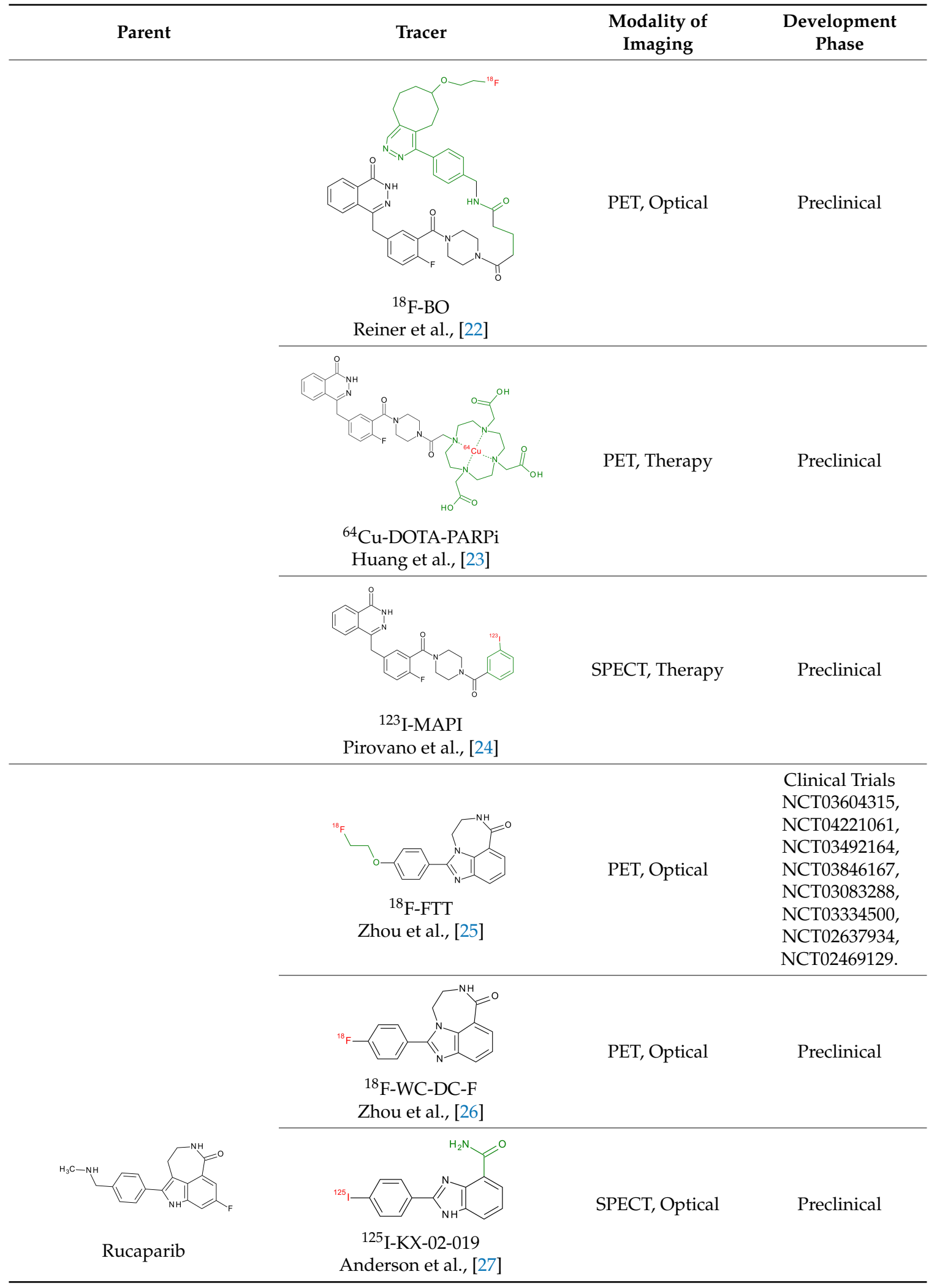


Table 1. Cont.

Parent

Due to the predominant nuclear target localization, PARPis usually diffuse passively across the plasma and nuclear membranes. For optimal passage across lipid bilayers, lipophilicity of Log P: 1.5-3.0 is optimal [31]. A further increase in lipophilicity ( $\left.\log \mathrm{P}_{\mathrm{oct}}>3.0\right)$ decreases passive diffusion across biological membranes, leading to low signal-to-noise ratios and hence is disadvantageous. Together with this, low dissociation constants $\left(\mathrm{K}_{\mathrm{i}}=1.2 \mathrm{nM}-5 \mathrm{nM}\right)$ prevent passive diffusion of PARPis out of the nucleus, avoiding quick wash-out [32]. Similarly, high \%PPB (>95\%) reduces the tissue penetration ability, and thus decreases the drug uptake by organs, and would require a higher dosage application [15]. In the case of brain malignancies like glioblastoma multiforme (GBM), the penetration of the blood-brain barrier (BBB) is essential for imaging and therapy. For this, the optimal drug parameters are $\log \mathrm{P}_{\text {oct }}: 2-3, \mathrm{MW}<450 \mathrm{Da}$, and PPB $<95 \%$ [15]. PARPis have shown different BBB penetration abilities in preclinical studies. While talazoparib, olaparib, and rucaparib show limited penetration across intact BBB as they are liable to efflux by the BBB, veliparib and niraparib were shown to have better penetration [33,34]. However, results from a Phase I clinical trial (OPARATIC trial) showed that olaparib was able to accumulate in marginal and core tumors in GBM patients who were treated with low doses of temozolomide [35].

Tracers with favorable in vitro characteristics are then investigated for in vivo biodistribution. In vivo, blood half-life, stability, tumor targeting, and PARP1 specificity are essential parameters to understand the tracer behavior, its suitability to help delineate tumor vs. non-tumor tissue, and to identify targeting efficiency. It should be taken into consideration that biologically defined in vivo parameters like vascular permeability, tumor microenvironment, and cellular composition further impact the drug concentration and by this the efficacy of PARP-targeted therapeutics.

Taken together, all these parameters play a critical role to define the properties of a diagnostic or theranostic agent at the cellular as well as the systemic level (i.e., drug delivery to tumor tissue). Hence, optimal in vivo pharmacokinetics and related parameters are essential for clinical translation of PARP theranostics. 


\section{Imaging with PARP-Addressing Tracers}

\subsection{Radio-fluorinated PARP Tracers}

The PET radionuclide ${ }^{18} \mathrm{~F}$ (half-life/ $\mathrm{T}_{1 / 2}=109.771 \mathrm{~min}$ ) is one of the most favored diagnostic radionuclides in PARP imaging probes. ${ }^{18} \mathrm{~F}$ conjugated PARPis like olaparib ( ${ }^{18} \mathrm{~F}$-Olaparib) [17], olaparib derivatives $\left({ }^{18} \mathrm{~F}-20\right.$ [15], $\left.{ }^{18} \mathrm{~F}-\mathrm{PARPi}[16]\right)$, and rucaparib derivatives $\left({ }^{18} \mathrm{~F}-\mathrm{Fluorthanatrace}\right.$ $\left({ }^{18}\right.$ F-FTT) [25], ${ }^{18}$ F-WC-DZ-F [26]) have been evaluated for imaging of PARP1 expression.

The most recently published PARP1 tracer is an ${ }^{18} \mathrm{~F}$-radiolabeled direct analog of olaparib $\left({ }^{18} \mathrm{~F}-\mathrm{Olaparib}\right)$ that has caught significant attention [17]. Even though a low radiochemical activity yield of $18 \% \pm 3 \%$ may be discouraging, its identical chemical composition to olaparib distinguishes it from other diagnostic tracers that are under investigation. Since it has similar pharmacokinetic and pharmacodynamic properties as its parental drug, apart from its use as a PARP1 targeting tracer, it can also provide insights into the systemic behavior of olaparib with regard to tumor accumulation and therapeutic dosage. Initial in vitro characterization using blocking studies in PSN-1, MiaPaCa-2, and Capan-1 cell lines showed in vitro PARP1 specificity. In a subcutaneous pancreatic ductal adenocarcinoma model, in vivo tumor uptake was enhanced upon radiation, confirming radiation-induced PARP1 overexpression. Recently Reilly et al., developed ${ }^{18} \mathrm{~F}-9 \mathrm{e}$ derived from the olaparib derivative AZD2461, for PARP1 imaging in neurodegenerative diseases. However, in spite of high PARP1 affinity $\left(\mathrm{IC}_{50}=3.9 \pm 1.2 \mathrm{nM}, \log \mathrm{P}=2.26\right)$, the tracer was seen to be impenetrable across the $\mathrm{BBB}$ both in rodents and in primate models, which suggests the lack of BBB penetration ability of the parental drug [21].

Previously, ${ }^{18} \mathrm{~F}$-conjugated tracers were developed as olaparib derivatives $\left({ }^{18} \mathrm{~F}-\mathrm{PARPi}\right.$ and $\left.{ }^{18} \mathrm{~F}-20\right)$ and characterized in GBM models. Both tracers have similar structures: ${ }^{18} \mathrm{~F}-\mathrm{PARPi}$ and ${ }^{18} \mathrm{~F}-20$ have fluorobenzamide and methylfluorobenzamide moieties respectively in place of the isopropyl moiety of olaparib [15,16]. Recently, a simplified process for the synthesis of ${ }^{18} \mathrm{~F}-\mathrm{PARPi}$ has been reported, reducing the synthesis time from $90 \mathrm{~min}$ to $66 \mathrm{~min}$, although the radiochemical yield obtained was $9.6 \%$ compared to $10 \%$ reported earlier [36]. Both ${ }^{18} \mathrm{~F}-\mathrm{PARPi}$ and ${ }^{18} \mathrm{~F}-20$ have been shown in subcutaneous models promising tumor-to-muscle ratios of $5.1 \pm 0.9$ and $3.6 \pm 0.5$, respectively. While ${ }^{18} \mathrm{~F}$-PARPi showed an encouraging tumor-to-brain ratio of $54.9 \pm 14.1$ (orthotopic model). ${ }^{18} \mathrm{~F}-20$ suffered from heavy defluorination $(>8.5 \% \mathrm{ID} / \mathrm{g}$ bone uptake, $1 \mathrm{~h}$ p.i), preventing it from further investigation [16]. In further preclinical studies, ${ }^{18} \mathrm{~F}$-PARPi was used to quantify target engagement of clinical PARPis (olaparib and talazoparib) in Non-Small Cell Lung Cancer (NSCLC) models upon co-treatment. This study enabled deciphering dosage regimens for complete drug-target engagement (olaparib: $15 \mathrm{mg} / \mathrm{kg}$; talazoparib $-5 \mathrm{mg} / \mathrm{kg}$ ) and tumor residence times (half-lives: olaparib-9.4 h; talazoparib-9.8 h) [37]. It was also used to monitor the target engagement of talazoparib where therapeutic and subtherapeutic dosage was distinguishable by differences in ${ }^{18} \mathrm{~F}-\mathrm{PARPi}$ uptake [38]. ${ }^{18} \mathrm{~F}$-PARPi was also studied as an alternative to ${ }^{18} \mathrm{~F}$-FDG for the delineation of oral cancer tissue from surrounding healthy tissue [39]. Furthermore, ${ }^{18} \mathrm{~F}-\mathrm{PARPi}$ has also proved to better differentiate radiation necrosis from tumors compared to ${ }^{18} \mathrm{~F}-\mathrm{FET}$ (in GBM models), and malignant from inflamed lymph nodes in B-cell lymphoma models [40,41].

Apart from olaparib derivatives, a rucaparib derivative, ${ }^{18} \mathrm{~F}-\mathrm{WC}-\mathrm{DZ}-\mathrm{F}$ has been characterized in a subcutaneous prostate cancer model [26]. Structurally, it is an analogue of ${ }^{18} \mathrm{~F}-\mathrm{FTT}$, which is currently in clinical trials. ${ }^{18} \mathrm{~F}-\mathrm{WC}-\mathrm{DZ}-\mathrm{F}$ was developed by replacing the ${ }^{125} \mathrm{I}$ in ${ }^{125} \mathrm{I}-\mathrm{KX} 1$ with ${ }^{18} \mathrm{~F}$, in order to improve the pharmacokinetics, in vivo tumor uptake and enhance blood stability. The tumor uptake was close to $4 \% \mathrm{ID} / \mathrm{c} . c$. ( $2 \mathrm{~h}$ p.i) as detected by PET imaging. However, ex vivo biodistribution data from naive mice showed unspecific uptake in tissues such as bone and muscle. Additionally, since TBRs and correlation with PARP1 expression levels were not reported, further studies will be needed to validate this tracer.

Other olaparib-derived ${ }^{18} \mathrm{~F}$ - tracers $\left({ }^{18} \mathrm{~F}-\mathrm{FTT},{ }^{18} \mathrm{~F}\right.$-BO and the dual modality PET/fluorescent imaging agent $\left.{ }^{18} \mathrm{~F}-\mathrm{PARPi}-\mathrm{FL}\right)$ have also been investigated. As they were already reviewed 
elaborately by previous reviews, they are spared from detailed discussion in this article to avoid redundancy $[4,20,22,42]$. Briefly, ${ }^{18} \mathrm{~F}-\mathrm{BO}$ has been tested in ovarian, breast, and pancreatic cancer models where uptake correlated with PARP1 expression. ${ }^{18} \mathrm{~F}-\mathrm{PARPi}-\mathrm{FL}$ showed higher specificity. However, it showed heavy in vivo defluorination ( $>10 \% \mathrm{ID} / \mathrm{g}$ bone uptake). ${ }^{18} \mathrm{~F}-\mathrm{FTT}$ was first validated in a subcutaneous breast cancer model showing promising tumor uptake $(4 \% \mathrm{ID} / \mathrm{cc}, 1 \mathrm{~h} \mathrm{p}$.i) [25]. It was also successfully validated in vitro and in vivo breast cancer models to image PARP1 expression levels and has progressed to clinical trials [43].

Besides PARPi-derived tracers, a substrate-based tracer $\left({ }^{18} \mathrm{~F}\right.$-labelled NAD analog), named ${ }^{18} \mathrm{~F}-$ Substrate-based PARP Activity Radiotracer ( ${ }^{18} \mathrm{~F}$-SuPAR) has been developed for imaging PARP-1/2 activity. The $\mathrm{N}_{6}$ of the adenine moiety in NAD is substituted with fluorinated poly-ethylene glycol $\left(\mathrm{F}-\mathrm{PEG}_{2}\right)$ prosthetic groups. Blocking experiments showed significant uptake reduction in an orthotopic breast cancer model but not in a subcutaneous model. However, correlation of ${ }^{18} \mathrm{~F}-S u P A R$ accumulation and PAR levels in tumor sections proved the tracer specificity. PET images post external beam irradiation showed an increase in tumor uptake. The major disadvantage is that, although the modification of $\mathrm{NAD}^{+}$is optimized for PARP1/2 uptake, $\mathrm{NAD}^{+}$is not a PARP1-specific substrate, as seen in vitro and in vivo by the background uptake possibly by other oxidoreductase enzymes. Moreover, rapid clearance and low serum stability $\left(\mathrm{T}_{1 / 2}<60 \mathrm{~min}\right)$ are other hindrances for its use as a PARP1 imaging agent [30].

While the number of investigations on synthesizing and optimizing new PARP1 tracers are fast growing, two tracers, ${ }^{18} \mathrm{~F}-\mathrm{PARPi}$ and ${ }^{18} \mathrm{~F}-\mathrm{FTT}$, have now progressed to Phase I clinical trials.

There are currently two Phase I clinical trials related to ${ }^{18} \mathrm{~F}-\mathrm{PARPi}$. In a head and neck cancer imaging trial (NCT03631017), ${ }^{18} \mathrm{~F}-\mathrm{PARPi}$ administration was safe and well tolerated. It was shown that all ${ }^{18} \mathrm{~F}$-FDG avid lesions also showed ${ }^{18} \mathrm{~F}$-PARPi uptake with comparable contrast ratios. Interestingly, several lymph nodes that were ${ }^{18} \mathrm{~F}-\mathrm{PARPi}$, but not ${ }^{18} \mathrm{~F}-\mathrm{FDG}$ avid, resolved after chemoradiation [44]. In a second, ongoing clinical trial, ${ }^{18} \mathrm{~F}-\mathrm{PARPi}$ is investigated for imaging of brain tumors (NCT04173104).

The other PARP1 tracer undergoing clinical evaluation is ${ }^{18}$ F-FTT. Several trials are ongoing, which are listed in ClinicalTrials.org to study ${ }^{18} \mathrm{~F}-\mathrm{FTT}$ as a PARP1 tracer pre-/post-treatment, in a wide range of cancers like ovarian (NCT03604315, NCT02637934), breast (NCT03846167), pancreatic (NCT03492164), prostate (NCT03334500), and GBM patients (NCT04221061). The first in-human trials showed promising uptake by tumor tissue in a cholangiocarcinoma patient [45]. Recently, a Phase I trial report using ${ }^{18} \mathrm{~F}$-FTT in a cohort of ovarian cancer patients pre-treated with chemotherapy showed discernible tumor uptake, inter-tumor heterogeneity, and positive correlation between high ${ }^{18} \mathrm{~F}-\mathrm{FTT}$ uptake and platinum-treatment resistance (Figure 2). Moreover, the study reports no correlation between uptake of ${ }^{18} \mathrm{~F}$-FDG and ${ }^{18} \mathrm{~F}$-FTT. ${ }^{18} \mathrm{~F}$-FDG and ${ }^{18} \mathrm{~F}$-FTT were shown to give complementary information enabling detection of metastatic omental lesions. Immunohistochemistry of clinical specimens showed PARP1 overexpression in lymph nodes with and without nodal disease. As a result, an accurate differentiation between malignant and reactive/inflammatory lymph nodes was not possible. Even though the study mentions high ${ }^{18}$ F-FDG and low ${ }^{18}$ F-FTT uptake in one patient having inflammatory lymph nodes, further investigation is required with a larger cohort [46].

\subsection{Radioiodinated PARP1 Tracers}

PARP inhibitors labeled with different PET/SPECT radioisotopes of iodine (123/124/125/131 I), have also been developed initially as imaging tracers and furthermore, their theranostic efficacy was explored. Since Iodine has a larger molecular weight, the pharmacokinetics of radio-iodinated tracers vary greatly from the parent drug. In 2015, two independent studies reported the synthesis of iodinated olaparib derivatives. Both studies characterized the same tracer backbone structure using different synthesis protocols and labelled with different radioisotopes of iodine $\left({ }^{123 / 125} \mathrm{I}\right.$ and $\left.{ }^{131 / 124} \mathrm{I} 2-\mathrm{PARP}\right)[18,19]$. 


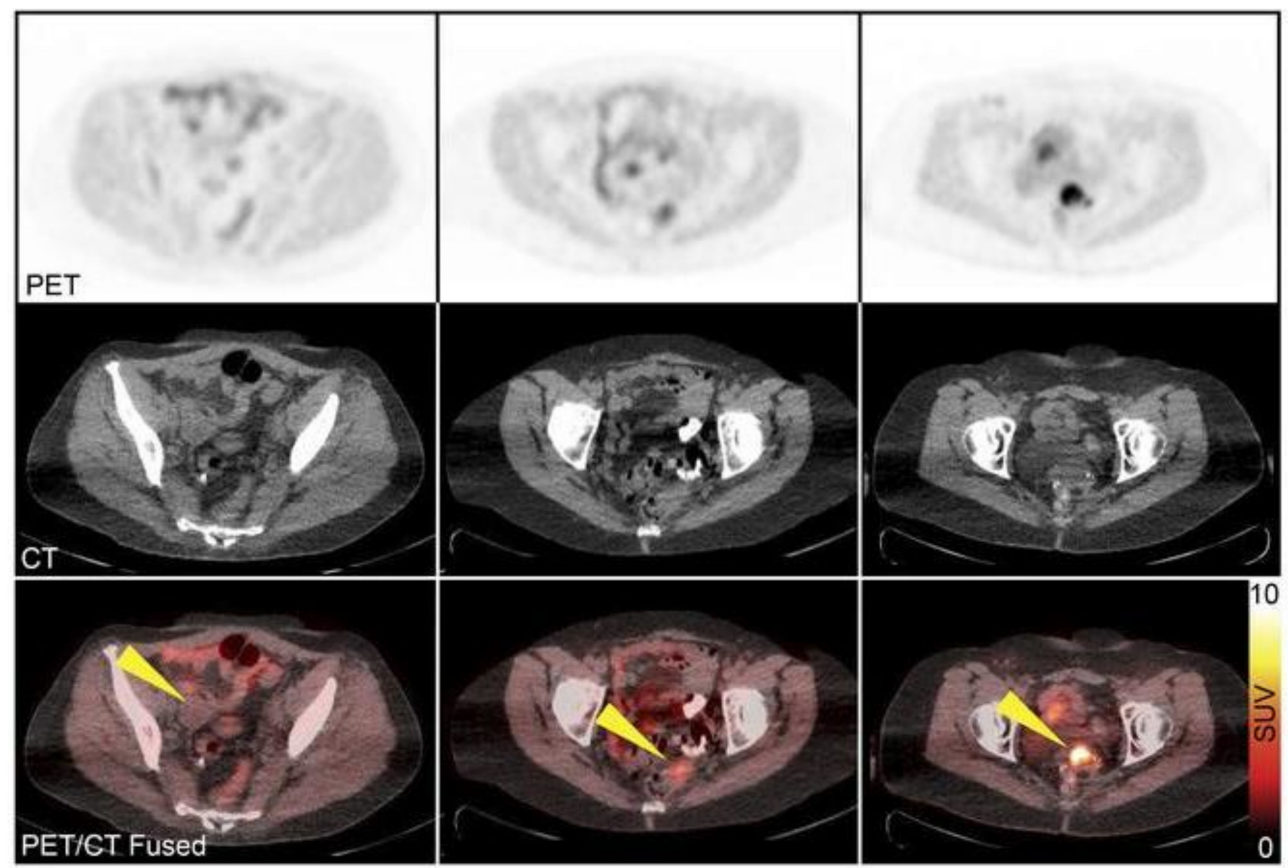

Figure 2. PET/CT images of ${ }^{18} \mathrm{~F}-\mathrm{FTT}$ uptake in ovarian cancer patients. PET/CT images from a clinical trial (NCT02637934) in three ovarian cancer patients show a wide range of ${ }^{18} \mathrm{~F}$-FTT uptake in tumor lesions. Standard uptake value (SUV) ranges from 2 (top-left) to 12 (top-right). Yellow arrows indicate sites of tumor. Reproduced with permission from Makvandi et al., titled "A PET imaging agent to evaluate PARP expression in ovarian cancer", published by The Journal of Clinical Investigation, 2018 [46].

In the report by Salinas et al., ${ }^{131 / 124} \mathrm{I} 2-\mathrm{PARPi}\left(\mathrm{T}_{1 / 2}=8.01 \mathrm{~d}\right.$ for ${ }^{131} \mathrm{I} ; 4.17 \mathrm{~d}$ for $\left.{ }^{124} \mathrm{I}\right)$ was characterized and optimized as a potential PET/SPECT tracer in GBM models. Biodistribution at $2.5 \mathrm{~h}$ post i.v injection showed a remarkable tumor-to-brain ratio of $40.0 \pm 6.3$, and a tumor-to-muscle ratio of $13.7 \pm 4.1$, confirming tumor targeting and retention. Unexpectedly, in their biodistribution studies with a subcutaneous model, there was no direct correlation between an increase in specific activity of the administered tracer with an increase in tumor-to-muscle ratio. But heavy deiodination was seen, as the tumor-to-thyroid ratio was $1.82 \pm 0.25$, in spite of prior thyroid blocking with sodium iodide (NaI). This is possibly due to the tracer oxidation in the liver. Notably, tumor uptake at $2 \mathrm{~h}$ in a U87 subcutaneous model was reported as $0.17 \% \mathrm{ID} / \mathrm{g}$ (tumor: muscle $=\sim 4.36$ ) whereas in the U251 orthotopic model it was $0.43 \% \mathrm{ID} / \mathrm{g}$ (tumor: muscle $=13.7 \pm 4.1$ ) despite the same PARP1 expression levels in both U87 and U251 tissues. This can be explained by a possible disruption in the BBB of the orthotopic model, improving passive targeting to the brain [18].

Similarly, Zmuda et al. reported conjugation of SPECT radionuclides ${ }^{123 / 125} \mathrm{I},\left(\mathrm{T}_{1 / 2}=13.22 \mathrm{~h}\right.$ for ${ }^{123} \mathrm{I} ; 59.49 \mathrm{~d}$ for ${ }^{125} \mathrm{I}$ ) to the same precursor and similar coupling conditions as Salinas et al. The biodistribution in a subcutaneous GBM model showed a TBR similar to that of the earlier reported tracer. Even though high plasma protein binding (96.2\%) is tolerable for imaging of primary tumor due to BBB disruptions, it is not optimal for PARP1 imaging of metastasis with an intact BBB [19].

These two studies were the first reports on radioiodine tagging as a convincing imaging strategy for PARP1 expression.

In 2016, two studies reported the synthesis and biodistribution of radio-iodinated benzimidazole PARPi (AG14032) derivatives ( ${ }^{125} \mathrm{I}-\mathrm{KX}-02-019$ and $\left.{ }^{125} \mathrm{I}-\mathrm{KX} 1\right)$, analogous of ${ }^{18} \mathrm{~F}$-FTT $[27,28]$. Though ${ }^{125} \mathrm{I}-\mathrm{KX} 1$ showed high tumor uptake ( $5 \% \mathrm{ID} / \mathrm{g}$ at $2 \mathrm{~h}$ p.i), olaparib pre-injection (i.p.) did not reduce tracer accumulation in the tumor, which is the standard way to evaluate PARP specificity. Furthermore, biodistribution of ${ }^{125} \mathrm{I}-\mathrm{KX}-02-019$ showed heavy deiodination (Thyroid uptake $\sim 5 \% \mathrm{ID} / \mathrm{g}$ vs. tumor uptake $\sim 1 \% \mathrm{ID} / \mathrm{g}$ ) at $4 \mathrm{~h}$ p.i. 


\subsection{PARP1 Targeted Theranostics}

Most recently, theranostic PARP radio-ligands have been developed. Here, therapeutic radionuclides, i.e., $\alpha, \beta^{-}$, and auger emitters, were conjugated to PARPis with the goal of effectively inflicting DNA damage on cancer cells, as binding to PARP1 leads to radioactive decay events in close proximity of the DNA. Importantly, these radionuclides also emit either positrons or $\gamma$-rays and hence allow evaluation of the tracer's in vivo behavior via PET/SPECT imaging. For theranostic PARP tracers, their therapeutic effect is not mitigated by PARP inhibition, but the PARP inhibitor acts as a delivery vehicle for the cytotoxic radiation. Theranostic radionuclides were either chosen for their short range, high linear energy transfer (LET) alpha $(\alpha)$ particle emissions (LET: $80-100 \mathrm{keV} / \mu \mathrm{m}$ upto $100 \mu \mathrm{m}$ ), auger electron emissions (LET $4-26 \mathrm{keV} / \mu \mathrm{m}$, upto $\sim 0.5 \mu \mathrm{m}$ ), or long range, low LET beta $(\beta)$ emissions (LET $\sim 0.2 \mathrm{keV} / \mu \mathrm{m}$, upto $1 \mathrm{~cm}$ ) [47].

\subsection{1. $\alpha$-emitter Theranostics}

Preclinical studies have been reported in neuroblastoma models with ${ }^{211} \mathrm{At}-\mathrm{MM} 4$, a rucaparib derivative (KX1) conjugated to an $\alpha$ - particle-emitting SPECT tracer ${ }^{211} \mathrm{At}\left(\mathrm{T}_{1 / 2}=7.5 \mathrm{~h}\right)$. Ex vivo biodistribution showed a rapid renal clearance, but tumor uptake increased from $\sim 1 \% \mathrm{ID} / \mathrm{g}$ at $2 \mathrm{~min} \mathrm{p} . \mathrm{i}$ to $14 \% \mathrm{ID} / \mathrm{g}$ at $2 \mathrm{~h}$ p.i. upon intravenous administration, with a promising tumor-to-blood ratio of 7.5. Therapeutic efficacy in mice showed a remarkable increase in median survival from $35 \mathrm{~d}$ (untreated) to $61 \mathrm{~d}$ (single dose) and even further to $80 \mathrm{~d}$ (multiple fractionated doses). These results are promising, especially as the animals showed no weight loss, no tumor regrowth, and minimal residual tumor at the end of $80 \mathrm{~d}$, indicating low systemic toxicity at efficacious doses [29].

\subsection{2. $\beta$ - emitter Theranostics}

The radionuclide ${ }^{64} \mathrm{Cu}\left(\mathrm{T}_{1 / 2}=12.7 \mathrm{~h}\right)$, which emits $\beta^{-}$particles for therapy and positrons for PET imaging was conjugated to DOTA-PARPi, derived from olaparib [23]. This tracer was characterized in mesothelioma models, with biodistribution showing peak tumor uptake at $1 \mathrm{~h} \mathrm{p.i}$ $(3.45 \pm 0.47 \% \mathrm{ID} / \mathrm{g})$, however tumor retention was poor as the tumor-to-muscle ratio at $18 \mathrm{~h}$ was $\sim 1$. Moreover, the conjugation of DOTA moiety reduced the binding affinity and thereby the cytotoxicity by 40 times. This would require an increase in the therapeutic dosage, which will in-turn increase systemic toxicity, and consequently limit its theranostic ability [23].

The earlier described ${ }^{131} \mathrm{I}$-PARPi can also be used as a theranostic compound, since ${ }^{131} \mathrm{I}$ is a $\gamma$ and $\beta^{-}$emitter. Jannetti et al. investigated its therapeutic efficacy in a subcutaneous GBM model [48]. Intratumorally administered fractionated doses (3x of $14.8 \mathrm{MBq}$ ) over $6 \mathrm{~d}$ slowed tumor growth, increasing median survival from $20 \mathrm{~d}$ ("cold" ${ }^{127}$ I-PARPi treated) to $29 \mathrm{~d}\left({ }^{131}\right.$ I-PARPi treated). Two weeks after the last dose, tumor growth progression was observed and showed a linear growth rate similar to that of control (PBS) and "cold" ${ }^{127}$ I-PARPi-treated cohorts. This can be explained by limited on-target residence time and/or the half-life $\left(\mathrm{T}_{1 / 2}=8 \mathrm{~d}\right)$ of ${ }^{131} \mathrm{I}$. To mimic a Convection Enhanced Delivery (CED), an osmotic pump mediated delivery into brain was investigated. In this orthotopic model, feasibility of strongly increasing brain uptake in tumor mice compared to naïve mice was shown, although the tumor vs. healthy brain tissue uptakes (tumor-to-brain ratio) was not reported. Although intratumoral applications are used for targeted delivery into brain malignancies in clinical studies, tumor-brain delineation is critical to assess and avoid neurotoxicities. Therefore, careful precaution with dosage is needed in case of intratumoral application of ${ }^{131} \mathrm{I}$ in glioblastoma to avoid any risk of bystander effect on surrounding healthy brain tissues $[49,50]$.

\subsubsection{Auger Emitter Theranostics}

Use of auger emitters $\left({ }^{123 / 125} \mathrm{I}\right)$ in PARP theranostic agents was reported by Lee et al., with ${ }^{125} \mathrm{I}-\mathrm{KX} 1$. The cytotoxic efficiency in neuroblastoma cell lines treated with ${ }^{125} \mathrm{I}-\mathrm{KX} 1$ was $10^{4}-10^{6}$ times higher than its non-radioactive precursor KX1. DNA damage induction was significantly higher compared 
to veliparib treatment as measured by $\mathrm{pH} 2 \mathrm{AX}$ fluorescence intensity [51]. Its PARP1 specificity was demonstrated by Makvandi et al., where PARP1 KO ovarian cancer cell lines showed reduced ${ }^{125} \mathrm{I}-\mathrm{KX} 1$ uptake [46]. However, further in vivo survival studies are required to evaluate its anti-tumor efficiency.

Another auger-emitting theranostic tracer, ${ }^{123}$ I-MAPi (Iodine-123 Meitner-Auger PARP1 inhibitor), an isotopologue of ${ }^{131}$ I-PARPi, has been studied in GBM models [24]. Survival increased from 40d to $58 \mathrm{~d}$ upon intratumoral delivery. Intratumoral delivery to an orthotopic model (using an osmotic pump) showed a further prolonged survival to $72 \mathrm{~d}$. Of note, intrathecal delivery has reported to result in stress-related deaths in mice, which was attributed to the small volume of the murine skull, and hence should only be a limiting factor in small animal studies. These results support the note that auger emission-mediated anti-tumor efficiency is promising.

Further studies are required for a comparison of the therapeutic efficacies of the auger emitting theranostic probes ${ }^{125} \mathrm{I}-\mathrm{KX} 1$ and ${ }^{123} \mathrm{I}-\mathrm{MAPi}$.

In neuroblastoma 3D solid tumor models, comparison of the cytotoxic efficiencies of ${ }^{125} \mathrm{I}-\mathrm{KX} 1$ with other PARP1-targeted ( ${ }^{211}$ At-MM4) or non-PARP1-targeted $\left({ }^{125} \mathrm{I}-\mathrm{MIBG}\right)$ radiopharmaceuticals showed that ${ }^{125} \mathrm{I}-\mathrm{KX} 1$ was less effective compared to ${ }^{211} \mathrm{At}-\mathrm{MM} 4$ in terms of concentration, tumor dosage (350x lower per decay), and tumor-cell nuclei dosage (150x lower per decay) [51]. The theranostic ability of ${ }^{211} \mathrm{At}$ is comparably advantageous to ${ }^{125} \mathrm{I}$ due to its superior cytotoxicity, and thereby low dosage requirement in addition to its favorable physical half-life. Taken together, amongst the theranostic PARP tracers, ${ }^{211}$ At-MM4 has shown the most promising in vivo anti-tumor efficiency.

\section{Future Prospects and Conclusion}

PARP1 imaging is emerging as a novel tool for assessing PARP1 expression in tumors and monitoring PARP inhibitor therapy response in the clinic. The rising interest in this field is evident from the development of several tracers in a short time span. The PARP1 tracers discussed in this work have been evaluated in various cancer types, which all show PARP overexpression. In vivo tumor characterization facilitated by PARP1 imaging could become a valuable instrument for personalized therapy in patients, which is the need of the hour.

Among the tracers reported, ${ }^{18} \mathrm{~F}-\mathrm{FTT}$ and ${ }^{18} \mathrm{~F}$-PARPi have progressed to clinical trials showing promising results in ovarian cancer and head and neck cancer patients, respectively and are further investigated in several types of solid tumors. Upon reproducible validation in the future, ${ }^{18} \mathrm{~F}$-Olaparib could also be a potential candidate for clinical trials due to its chemical identity to olaparib. Some hurdles that lay before translation of other tracers include improving serum stability, optimizing tumor uptake, tackling in vivo dehalogenation, and avoiding off-target uptake.

For theranostics, primary challenges include optimizations on a) tumor residence time vs. absorbed dose, b) tumor vs. clearance-organ uptake, c) compromise between $T_{1 / 2}$ of the chosen radionuclide and its therapeutic efficiency. Among the reported theranostic compounds, the $\alpha$ emitting ${ }^{211} \mathrm{At}-\mathrm{MM} 4$ has shown encouraging anti-tumor effects. However, considerable cytotoxicity on clearance organs such as liver and stomach, as well as its susceptibility to de-astatination are persisting challenges. Moreover, patients pre-treated with platinum-based chemotherapy show elevated PARP1 levels in the tumor microenvironment. Though this can lead to an overestimation of tumor size by imaging tracers, the high PARP1 levels in the tumor microenvironment can enhance theranostic tracer uptake leading to better therapeutic efficacy.

Particularly for GBM, crossing the BBB is a roadblock for small molecule drugs including most PARP inhibitors. Feasibility of bypassing the BBB via intrathecal application or intratumoral delivery by CED was shown preclinically and could be an approach to efficiently deliver theranostic PARPi to brain malignancies [52]. Nevertheless, alternative minimally invasive strategies (e.g., intravenous injection) to address brain and other malignancies should also be developed to minimize the risk of infection, neural toxicity, pain, and patient discomfort. For non-invasive tumor-targeted delivery, nanomedicines could be a possible solution $[53,54]$. Recent preclinical studies in glioma models present nanoparticles developed using poly-MPC coating as an effective way to cross BBB $[55,56]$. Nano-formulations of 
PARPi have already been reported, although none with radiotracers. Nanoemulsion-based delivery of PARPi-FL showed increased blood half-life, and delineated subcutaneous xenografts of small cell lung cancer [57]. Liposomal talazoparib showed significant increase in survival and reduction in side effects [58]. Loading radiolabeled drugs in nanoparticles is challenging, but could overcome current limitations for PARP-targeted alpha and auger emitters, as this can potentially reduce off-target DNA damage by diminishing off-target uptake [59]. Nanoscale delivery systems have proven to minimize side-effects, for e.g., the in-use lysosomal doxorubicin (Doxilß) [60]. This calls for further research towards developing mechanisms for targeted delivery to tumors, which will improve their future prospects.

Taken together, PARP1 overexpression in various cancers can be exploited as a molecular target in the clinic. Imaging and therapy tracers have been developed with promising preclinical results and a number of relevant clinical applications have been outlined. Upon optimization, some tracers are fast approaching clinical translation.

Author Contributions: Conceptualization, writing—original draft preparation R.A.S.; writing—review and editing, S.K., W.W., A.M., M.B. and F.M.M. All authors have read and agreed to the published version of the manuscript.

Funding: This work was supported by the Deutsche Forschungsgemeinschaft (DFG) in the framework of the Research Training Group ,Tumor-targeted Drug Delivery" grant 331065168. FMM received research funding from the ITN INTRICARE of European Union's Horizon 2020 research and innovation program under the Marie Sklodowska Curie (grant 722609).

Acknowledgments: In this section you can acknowledge any support given which is not covered by the author contribution or funding sections. This may include administrative and technical support, or donations in kind (e.g., materials used for experiments).

Conflicts of Interest: The authors declare no conflict of interest.

\section{Abbreviations}

$\begin{array}{ll}\text { PARP1 } & \text { Poly ADP Ribose Polymerase 1 } \\ \text { NAD } & \text { Nicotinamide Adenine Dinucleotide } \\ \text { PPB } & \text { Plasma protein binding } \\ \text { GBM } & \text { Glioblastoma multiforme } \\ \text { BBB } & \text { Blood-brain barrier } \\ \text { FDG } & \text { Fluorodeoxyglucose } \\ \text { DOTA } & \text { Dodecane Tetraacetic acid }\end{array}$

\section{References}

1. Chatterjee, N.; Walker, G.C. Mechanisms of DNA damage, repair, and mutagenesis. Environ. Mol. Mutagen. 2017, 58, 235-263. [CrossRef] [PubMed]

2. Ray Chaudhuri, A.; Nussenzweig, A. The multifaceted roles of PARP1 in DNA repair and chromatin remodelling. Nat. Rev. Mol. Cell Biol. 2017, 18, 610-621. [CrossRef] [PubMed]

3. Javle, M.; Curtin, N.J. The role of PARP in DNA repair and its therapeutic exploitation. Br. J. Cancer 2011, 105, 1114-1122. [CrossRef] [PubMed]

4. Knight, J.C.; Koustoulidou, S.; Cornelissen, B. Imaging the DNA damage response with PET and SPECT. Eur. J. Nucl. Med. Mol. Imaging 2017, 44, 1065-1078. [CrossRef]

5. Kim, G.; Ison, G.; McKee, A.E.; Zhang, H.; Tang, S.; Gwise, T.; Sridhara, R.; Lee, E.; Tzou, A.; Philip, R.; et al. FDA approval summary: Olaparib monotherapy in patients with deleterious germline BRCA-mutated advanced ovarian cancer treated with three or more lines of chemotherapy. Clin. Cancer Res. 2015, 21, 4257-4261. [CrossRef]

6. Balasubramaniam, S.; Beaver, J.A.; Horton, S.; Fernandes, L.L.; Tang, S.; Horne, H.N.; Liu, J.; Liu, C.; Schrieber, S.J.; Yu, J.; et al. FDA approval summary: Rucaparib for the treatment of patients with deleterious BRCA mutation-associated advanced ovarian cancer. Clin. Cancer Res. 2017, 23, 7165-7170. [CrossRef] 
7. Ison, G.; Howie, L.J.; Amiri-Kordestani, L.; Zhang, L.; Tang, S.; Sridhara, R.; Pierre, V.; Charlab, R.; Ramamoorthy, A.; Song, P.; et al. FDA approval summary: Niraparib for the maintenance treatment of patients with recurrent ovarian cancer in response to platinum-based chemotherapy. Clin. Cancer Res. 2018, 24, 4066-4071. [CrossRef]

8. Hoy, S.M. Talazoparib: First Global Approval. Drugs 2018, 78, 1939-1946. [CrossRef]

9. Lord, C.J.; Ashworth, A. PARP inhibitors: Synthetic lethality in the clinic. Science 2017, 355, 1152-1158. [CrossRef]

10. Del Conte, G.; Sessa, C.; von Moos, R.; Viganò, L.; Digena, T.; Locatelli, A.; Gallerani, E.; Fasolo, A.; Tessari, A.; Cathomas, R.; et al. Phase I study of olaparib in combination with liposomal doxorubicin in patients with advanced solid tumours. Br. J. Cancer 2014, 111, 651-659. [CrossRef]

11. Hinchcliff, E.; Westin, S.N.; Dal Molin, G.; Lafargue, C.J.; Coleman, R.L. Poly-ADP-ribose polymerase inhibitor use in ovarian cancer: Expanding indications and novel combination strategies. Int. J. Gynecol. Cancer 2019, 29, 956-968. [CrossRef] [PubMed]

12. Kossatz, S.; Weber, W.; Reiner, T. Detection and Delineation of Oral Cancer with a PARP1-Targeted Optical Imaging Agent. Mol. Imaging 2017, 16, 1536012117723786. [CrossRef] [PubMed]

13. Kossatz, S.; Pirovano, G.; Demétrio De Souza França, P.; Strome, A.L.; Sunny, S.P.; Zanoni, D.K.; Mauguen, A.; Carney, B.; Brand, C.; Shah, V.; et al. Validation of the use of a fluorescent PARP1 inhibitor for the detection of oral, oropharyngeal and oesophageal epithelial cancers. Nat. Biomed. Eng. 2020, 4, 272-285. [CrossRef] [PubMed]

14. Adam, M.J.; Wilbur, D.S. Radiohalogens for imaging and therapy. Chem. Soc. Rev. 2005, 34, $153-163$. [CrossRef]

15. Zmuda, F.; Blair, A.; Liuzzi, M.C.; Malviya, G.; Chalmers, A.J.; Lewis, D.; Sutherland, A.; Pimlott, S.L. An ${ }^{18}$ F-Labeled Poly(ADP-ribose) Polymerase Positron Emission Tomography Imaging Agent. J. Med. Chem. 2018, 61, 4103-4114. [CrossRef]

16. Carney, B.; Carlucci, G.; Salinas, B.; Di Gialleonardo, V.; Kossatz, S.; Vansteene, A.; Longo, V.A.; Bolaender, A.; Chiosis, G.; Keshari, K.R.; et al. Non-invasive PET Imaging of PARP1 Expression in Glioblastoma Models. Mol. Imaging Biol. 2016, 18, 386-392. [CrossRef] [PubMed]

17. Chen, D.L.; Wilson, T.C.; Xavier, M.-A.; Knight, J.; Verhoog, S.; Torres, J.B.; Mosley, M.; Hopkins, S.L.; Wallington, S.; Allen, P.D.; et al. PET Imaging of PARP Expression Using ${ }^{18}$ F-Olaparib. J. Nucl. Med. 2019, 60, 504-510. [CrossRef]

18. Salinas, B.; Irwin, C.P.; Kossatz, S.; Bolaender, A.; Chiosis, G.; Pillarsetty, N.; Weber, W.A.; Reiner, T. Radioiodinated PARP1 tracers for glioblastoma imaging. EJNMMI Res. 2015, 5, 46. [CrossRef]

19. Zmuda, F.; Malviya, G.; Blair, A.; Boyd, M.; Chalmers, A.J.; Sutherland, A.; Pimlott, S.L. Synthesis and Evaluation of a Radioiodinated Tracer with Specificity for Poly(ADP-ribose) Polymerase-1 (PARP-1) in vivo. J. Med. Chem. 2015, 58, 8683-8693. [CrossRef]

20. Keliher, E.J.; Klubnick, J.A.; Reiner, T.; Mazitschek, R.; Weissleder, R. Efficient acid-catalyzed ${ }^{18} \mathrm{~F} /{ }^{19} \mathrm{~F}$ fluoride exchange of BODIPY dyes. ChemMedChem 2014, 9, 1368-1373. [CrossRef]

21. Reilly, S.W.; Puentes, L.N.; Schmitz, A.; Hsieh, C.-J.; Weng, C.-C.; Hou, C.; Li, S.; Kuo, Y.-M.; Padakanti, P.; Lee, H.; et al. Synthesis and evaluation of an AZD2461 [ $\left.{ }^{18} \mathrm{~F}\right]$ PET probe in non-human primates reveals the PARP-1 inhibitor to be non-blood-brain barrier penetrant. Bioorgan. Chem. 2019, 83, 242-249. [CrossRef] [PubMed]

22. Reiner, T.; Keliher, E.J.; Earley, S.; Marinelli, B.; Weissleder, R. Synthesis and in vivo imaging of a ${ }^{18}$ F-labeled PARP1 inhibitor using a chemically orthogonal scavenger-assisted high-performance method. Angew. Chem. Int. Ed. Engl. 2011, 50, 1922-1925. [CrossRef] [PubMed]

23. Huang, T.; Hu, P.; Banizs, A.B.; He, J. Initial evaluation of Cu-64 labeled PARPi-DOTA PET imaging in mice with mesothelioma. Bioorgan. Med. Chem. Lett. 2017, 27, 3472-3476. [CrossRef] [PubMed]

24. Pirovano, G.; Jannetti, S.A.; Carter, L.M.; Sadique, A.; Kossatz, S.; Guru, N.; Demétrio De Souza França, P.; Maeda, M.; Zeglis, B.M.; Lewis, J.S.; et al. Targeted brain tumor radiotherapy using an Auger emitter. Clin. Cancer Res. 2020. [CrossRef]

25. Zhou, D.; Chu, W.; Xu, J.; Jones, L.A.; Peng, X.; Li, S.; Chen, D.L.; Mach, R.H. Synthesis, $\left[{ }^{18}\right.$ F] radiolabeling, and evaluation of poly (ADP-ribose) polymerase-1 (PARP-1) inhibitors for in vivo imaging of PARP-1 using positron emission tomography. Bioorgan. Med. Chem. 2014, 22, 1700-1707. [CrossRef] 
26. Zhou, D.; Xu, J.; Mpoy, C.; Chu, W.; Kim, S.H.; Li, H.; Rogers, B.E.; Katzenellenbogen, J.A. Preliminary evaluation of a novel ${ }^{18}$ F-labeled PARP-1 ligand for PET imaging of PARP-1 expression in prostate cancer. Nucl. Med. Biol. 2018, 66, 26-31. [CrossRef]

27. Anderson, R.-C.; Makvandi, M.; Xu, K.; Lieberman, B.P.; Zeng, C.; Pryma, D.A.; Mach, R.H. Iodinated benzimidazole PARP radiotracer for evaluating PARP1/2 expression in vitro and in vivo. Nucl. Med. Biol. 2016, 43, 752-758. [CrossRef]

28. Makvandi, M.; Xu, K.; Lieberman, B.P.; Anderson, R.-C.; Effron, S.S.; Winters, H.D.; Zeng, C.; McDonald, E.S.; Pryma, D.A.; Greenberg, R.A.; et al. A Radiotracer Strategy to Quantify PARP-1 Expression in vivo Provides a Biomarker That Can Enable Patient Selection for PARP Inhibitor Therapy. Cancer Res. 2016, 76, 4516-4524. [CrossRef]

29. Makvandi, M.; Lee, H.; Puentes, L.N.; Reilly, S.W.; Rathi, K.S.; Weng, C.-C.; Chan, H.S.; Hou, C.; Raman, P.; Martinez, D.; et al. Targeting PARP-1 with Alpha-Particles is Potently Cytotoxic to Human Neuroblastoma in Preclinical Models. Mol. Cancer Ther. 2019, 18, 1195-1204. [CrossRef]

30. Shuhendler, A.J.; Cui, L.; Chen, Z.; Shen, B.; Chen, M.; James, M.L.; Witney, T.H.; Bazalova-Carter, M.; Gambhir, S.S.; Chin, F.T.; et al. [ ${ }^{18}$ F]-SuPAR: A Radiofluorinated Probe for Noninvasive Imaging of DNA Damage-Dependent Poly(ADP-ribose) Polymerase Activity. Bioconjug. Chem. 2019, 30, 1331-1342. [CrossRef]

31. Jacobson, O.; Chen, X. Interrogating tumor metabolism and tumor microenvironments using molecular positron emission tomography imaging. Theranostic approaches to improve therapeutics. Pharmacol. Rev. 2013, 65, 1214-1256. [CrossRef]

32. Jiang, X.; Li, W.; Li, X.; Bai, H.; Zhang, Z. Current status and future prospects of PARP inhibitor clinical trials in ovarian cancer. Cancer Manag. Res. 2019, 11, 4371-4390. [CrossRef] [PubMed]

33. Gupta, S.K.; Smith, E.J.; Mladek, A.C.; Tian, S.; Decker, P.A.; Kizilbash, S.H.; Kitange, G.J.; Sarkaria, J.N. PARP Inhibitors for Sensitization of Alkylation Chemotherapy in Glioblastoma: Impact of Blood-Brain Barrier and Molecular Heterogeneity. Front. Oncol. 2019, 8, 670. [CrossRef] [PubMed]

34. Sun, K.; Mikule, K.; Wang, Z.; Poon, G.; Vaidyanathan, A.; Smith, G.; Zhang, Z.Y.; Hanke, J.; Ramaswamy, S.; Wang, J. A comparative pharmacokinetic study of PARP inhibitors demonstrates favorable properties for niraparib efficacy in preclinical tumor models. Oncotarget 2018, 9, 37080-37096. [CrossRef] [PubMed]

35. Hanna, C.; Kurian, K.M.; Williams, K.; Watts, C.; Jackson, A.; Carruthers, R.; Strathdee, K.; Cruickshank, G.; Dunn, L.; Erridge, S.; et al. Pharmacokinetics, safety and tolerability of olaparib and temozolomide for recurrent glioblastoma: Results of the phase I OPARATIC trial. Neuro Oncol. 2020, XX, 1-11. [CrossRef]

36. Wilson, T.C.; Pillarsetty, N.; Reiner, T. A One-Pot Radio-synthesis of [ ${ }^{18}$ F]PARPi. J. Label. Compd. Radiopharm. 2020, 1-7. [CrossRef]

37. Carney, B.; Kossatz, S.; Lok, B.H.; Schneeberger, V.; Gangangari, K.K.; Pillarsetty, N.V.K.; Weber, W.A.; Rudin, C.M.; Poirier, J.T.; Reiner, T. Target engagement imaging of PARP inhibitors in small-cell lung cancer. Nat. Commun. 2018, 9, 176. [CrossRef]

38. Laird, J.; Lok, B.H.; Carney, B.; Kossatz, S.; de Stanchina, E.; Reiner, T.; Poirier, J.T.; Rudin, C.M. Positron-Emission Tomographic Imaging of a Fluorine 18-Radiolabeled Poly(ADP-Ribose) Polymerase 1 Inhibitor Monitors the Therapeutic Efficacy of Talazoparib in SCLC Patient-Derived Xenografts. J. Thorac. Oncol. 2019, 14, 1743-1752. [CrossRef]

39. França, P.D.D.S.; Roberts, S.; Kossatz, S.; Guru, N.; Mason, C.; Zanoni, D.K.; Abrahão, M.; Schöder, H.; Ganly, I.; Patel, S.; et al. Fluorine-18 labeled poly (ADP-ribose) polymerase1 inhibitor as a potential alternative to 2-deoxy-2-[18 F]fluoro-d-glucose positron emission tomography in oral cancer imaging. Nucl. Med. Biol. 2020, 84, 80-87. [CrossRef]

40. Donabedian, P.L.; Kossatz, S.; Engelbach, J.A.; Jannetti, S.A.; Carney, B.; Young, R.J.; Weber, W.A.; Garbow, J.R.; Reiner, T. Discriminating radiation injury from recurrent tumor with $\left[{ }^{18} \mathrm{~F}\right] \mathrm{PARPi}$ and amino acid PET in mouse models. EJNMMI Res. 2018, 8, 59. [CrossRef]

41. Tang, J.; Salloum, D.; Carney, B.; Brand, C.; Kossatz, S.; Sadique, A.; Lewis, J.S.; Weber, W.A.; Wendel, H.G.; Reiner, T. Targeted PET imaging strategy to differentiate malignant from inflamed lymph nodes in diffuse large B-cell lymphoma. Proc. Natl. Acad. Sci. USA 2017, 114, E7441-E7449. [CrossRef] [PubMed]

42. Carney, B.; Kossatz, S.; Reiner, T. Molecular Imaging of PARP. J. Nucl. Med. 2017, 58, 1025-1030. [CrossRef] [PubMed] 
43. Edmonds, C.E.; Makvandi, M.; Lieberman, B.P.; Xu, K.; Zeng, C.; Li, S.; Hou, C.; Lee, H.; Greenberg, R.A.; Mankoff, D.A.; et al. $\left[{ }^{18} \mathrm{~F}\right]$ FluorThanatrace uptake as a marker of PARP1 expression and activity in breast cancer. Am. J. Nucl. Med. Mol. Imaging 2016, 6, 94-101.

44. Schöder, H.M.; Demétrio De Souza França, P.; Nakajima, R.; Burnazi, E.M.; Roberts, S.; Brand, C.; Grkovski, M.; Mauguen, A.; Dunphy, M.P.; Ghossein, R.; et al. Safety and feasibility of PARP1/2 imaging with 18F-PARPi in patients with head and neck cancer. Clin. Cancer Res. 2020. [CrossRef] [PubMed]

45. Chen, D.; Dyroff, S.; Michel, L.; Wang-Gillam, A.; Tan, B.; Phillips, S.; Bognar, C.; Chu, W.; Zhou, D.; Mach, R.; et al. First-in-human studies characterizing a poly(ADP-ribose)polymerase (PARP) targeted tracer, 18F-FluorThanatrace (18F-FTT) for cancer imaging. Soc. Nucl. Med. 2016, 57, 582. [CrossRef]

46. Makvandi, M.; Pantel, A.; Schwartz, L.; Schubert, E.; Xu, K.; Hsieh, C.-J.J.; Hou, C.; Kim, H.; Weng, C.-C.C.; Winters, H.; et al. A PET imaging agent for evaluating PARP-1 expression in ovarian cancer. J. Clin. Invest. 2018, 128, 2116-2126. [CrossRef]

47. Kassis, A.I. Therapeutic Radionuclides: Biophysical and Radiobiologic Principles. Semin. Nucl. Med. 2008, 38, 358-366. [CrossRef]

48. Jannetti, S.A.; Carlucci, G.; Carney, B.; Kossatz, S.; Shenker, L.; Carter, L.M.; Salinas, B.; Brand, C.; Sadique, A.; Donabedian, P.L.; et al. PARP-1-Targeted Radiotherapy in Mouse Models of Glioblastoma. J. Nucl. Med. 2018, 59, 1225-1233. [CrossRef]

49. Brady, D.; O'Sullivan, J.M.; Prise, K.M. What is the role of the Bystander response in radionuclide therapies? Front. Oncol. 2013, 3, 215. [CrossRef]

50. Boyd, M.; Sorensen, A.; McCluskey, A.G.; Mairs, R.J. Radiation quality-dependent bystander effects elicited by targeted radionuclides. J. Pharm. Pharmacol. 2008, 60, 951-958. [CrossRef]

51. Lee, H.; Riad, A.; Martorano, P.; Mansfield, A.; Samanta, M.; Batra, V.; Mach, R.H.; Maris, J.M.; Pryma, D.A.; Makvandi, M. PARP-1-targeted Auger emitters display high-LET cytotoxic properties in vitro but show limited therapeutic utility in solid tumor models of human neuroblastoma. J. Nucl. Med. 2020, 61, 850-856. [CrossRef] [PubMed]

52. Jahangiri, A.; Chin, A.T.; Flanigan, P.M.; Chen, R.; Bankiewicz, K.; Aghi, M.K. Convection-enhanced delivery in glioblastoma: A review of preclinical and clinical studies. J. Neurosurg. 2017, 126, 191-200. [CrossRef] [PubMed]

53. Lam, F.C.; Morton, S.W.; Wyckoff, J.; Vu Han, T.L.; Hwang, M.K.; Maffa, A.; Balkanska-Sinclair, E.; Yaffe, M.B.; Floyd, S.R.; Hammond, P.T. Enhanced efficacy of combined temozolomide and bromodomain inhibitor therapy for gliomas using targeted nanoparticles. Nat. Commun. 2018, 9, 1-11. [CrossRef] [PubMed]

54. Jain, K.K. Nanobiotechnology-based strategies for crossing the blood-brain barrier. Nanomedicine 2012, 7, 1225-1233. [CrossRef]

55. Meng, X.; Zhao, Y.; Han, B.; Zha, C.; Zhang, Y.; Li, Z.; Wu, P.; Qi, T.; Jiang, C.; Liu, Y.; et al. Dual functionalized brain-targeting nanoinhibitors restrain temozolomide-resistant glioma via attenuating EGFR and MET signaling pathways. Nat. Commun. 2020, 11, 1-15. [CrossRef]

56. Wang, N.; Sun, P.; Lv, M.; Tong, G.; Jin, X.; Zhu, X. Mustard-inspired delivery shuttle for enhanced blood-brain barrier penetration and effective drug delivery in glioma therapy. Biomater. Sci. 2017, 5, 1041-1050. [CrossRef]

57. Gonzales, J.; Kossatz, S.; Roberts, S.; Pirovano, G.; Brand, C.; Pérez-Medina, C.; Donabedian, P.; de la Cruz, M.J.; Mulder, W.J.M.M.; Reiner, T. Nanoemulsion-Based Delivery of Fluorescent PARP Inhibitors in Mouse Models of Small Cell Lung Cancer. Bioconjug. Chem. 2018, 29, 3776-3782. [CrossRef]

58. Zhang, D.; Baldwin, P.; Leal, A.S.; Carapellucci, S.; Sridhar, S.; Liby, K.T. A nano-liposome formulation of the PARP inhibitor talazoparib enhances treatment efficacy and modulates immune cell populations in mammary tumors of BRCA-deficient mice. Theranostics 2019, 9, 6224-6238. [CrossRef]

59. Lin, X.; Xie, J.; Niu, G.; Zhang, F.; Gao, H.; Yang, M.; Quan, Q.; Aronova, M.A.; Zhang, G.; Lee, S.; et al. Chimeric ferritin nanocages for multiple function loading and multimodal imaging. Nano Lett. 2011, 11, 814-819. [CrossRef]

60. Barenholz, Y. Doxil ${ }^{\circledR}$ —The first FDA-approved nano-drug: Lessons learned. J. Control. Release 2012, 160, 117-134. [CrossRef]

(C) 2020 by the authors. Licensee MDPI, Basel, Switzerland. This article is an open access article distributed under the terms and conditions of the Creative Commons Attribution (CC BY) license (http://creativecommons.org/licenses/by/4.0/). 